\title{
A novel combination approach of human polyclonal IVIG and antibiotics against multidrug-resistant Gram-positive bacteria
}

\section{Mariam Madkour Sallam \\ Khaled Abou-Aisha \\ Mohamed El-Azizi}

Department of Microbiology, Immunology, and Biotechnology, Faculty of Pharmacy and Biotechnology, German University in Cairo, New Cairo City, Cairo, Egypt
This article was published in the following Dove Press journal:

Infection and Drug Resistance

8 December 2016

Number of times this article has been viewed

Background: Gram-positive bacteria, especially methicillin-resistant Staphylococcus aureus (MRSA) and enterococci, have shown a remarkable ability to develop resistance to antimicrobial agents.

Objective: We aimed to assess possible enhancement of the antimicrobial activity of vancomycin, amoxicillin, clarithromycin, and azithromycin by human polyclonal intravenous immunoglobulin G (IVIG) against 34 multidrug-resistant (MDR) bacterial isolates, including MRSA, Enterococcus faecium, and Enterococcus faecalis.

Materials and methods: Double combinations of the antibiotics with the IVIG were assessed by checkerboard assay, where the interaction was evaluated with respect to the minimum inhibitory concentration (MIC) of the antibiotics. The results of the checkerboard assay were verified in vitro using time-kill assay and in vivo using an invasive sepsis murine model.

Results: The checkerboard assay showed that IVIG enhanced the antimicrobial activity of amoxicillin and clarithromycin against isolates from the three groups of bacteria, which were resistant to the same antibiotics when tested in the absence of IVIG. The efficacy of vancomycin against $15 \%$ of the tested isolates was enhanced when it was combined with the antibodies. Antagonism was demonstrated in $47 \%$ of the E. faecalis isolates when clarithromycin was combined with the IVIG. Synergism was proved in the time-kill assay when amoxicillin was combined with the antibodies; meanwhile, antagonism was not demonstrated in all tested combinations, even in combinations that showed such response in checkerboard assay.

Conclusion: The suggested approach is promising and could be helpful to enhance the antimicrobial activity of not only effective antibiotics but also antibiotics that have been proven to be ineffective against MDR bacteria. To our knowledge, this combinatorial approach against MDR bacteria, such as MRSA and enterococci, has not been investigated before.

Keywords: human polyclonal IVIG, MRSA, vancomycin, amoxicillin, Enterococcus faecalis, Enterococcus faecium, nonconventional antimicrobials, multidrug resistance

\section{Introduction}

The rate of emergence of antibiotic resistance has increased dramatically, which leads to failure of treatment of life-threatening bacterial infections. Failure of the antibiotics to manage infections caused by multidrug-resistant (MDR) bacteria results in increase in mortality, morbidity, and costs due to longer stay in health care settings. ${ }^{1-4}$ Infections caused by MDR Gram-positive bacteria, particularly methicillin-resistant Staphylococcus aureus (MRSA) and vancomycin-resistant Enterococcus (VRE), are challenging to clinicians not only due to their resistance to conventional antibiotics but also due to the emergence of resistant strains to
Correspondence: Mohamed El-Azizi Department of Microbiology, Immunology, and Biotechnology, Faculty of Pharmacy and Biotechnology, German University in Cairo, New Cairo City, Main Entrance El-Tagamoa El-Khames, Postal Code II835, Cairo, Egypt

Tel +20 $227589990-8$

Fax +2022758 I04I

Email mohamed.el-azizi@guc.edu.eg 
new antibiotics such as daptomycin and linezolid. ${ }^{5}$ MRSA is the most common cause of septic shock and multiple organ failure. The outcomes of treatment of severe infections caused by MRSA with currently available antibiotics are often unsatisfactory. ${ }^{3,6}$ Enterococcus faecalis and Enterococcus faecium, on the other hand, are responsible for a wide variety of diseases. Among them, endocarditis and bloodstream infections are the most severe and therapeutically challenging. ${ }^{7}$ Enterococcal infections are the third common cause of nosocomial infection and the third common cause of bacteremia in the USA. ${ }^{8}$ Enterococci have the potential for resistance to virtually all clinically useful antibiotics. ${ }^{9}$

With the limited number of effective antibiotics and the small number of new antimicrobials that have been developed recently, the emergence of MDR Gram-positive pathogens has driven the need to find alternative therapeutic approaches with higher efficacy and less resistance developed by the pathogens.

Commercially pooled polyclonal human intravenous immunoglobulin G (IVIG) is a therapeutic preparation that is made of human polyclonal immunoglobulin $\mathrm{G}$ ( $\mathrm{IgG}$ ) from large pools of plasma obtained from 10,000-50,000 healthy donors. ${ }^{10}$ IVIG has been approved by the US Food and Drug Administration (FDA) in autoimmune disorders and in primary immunodeficiency. ${ }^{10}$ It has other off-label uses against infectious and noninfectious disorders. ${ }^{11-14}$

The broad-spectrum antimicrobial activity of IVIG is mediated through the immune modulation of the host humoral immunity and cellular immunity, particularly opsonization and phagocytic activation. ${ }^{6}$ Since the mechanisms of the antimicrobial action of the antibodies are different from those of the antibiotics, cross-resistance of antibiotic-resistant strains to IVIG never exists. ${ }^{11}$

The pool sizes of the IVIG guarantee wide spectrum of antibody specificities, including neutralizing antibodies for bacterial virulence factors such as streptococcal-super antigen, $\alpha$-hemolysin, and coagulase of MRSA. ${ }^{6,15,16}$ It would be possible that IVIG contains neutralizing antibodies for bacterial virulence factors responsible for antibiotic resistance. Thus, combining the IVIG with antibiotics would be a new strategy to combat serious infections caused by MDR bacteria.

The present investigation aimed to assess the possible enhancement of the antimicrobial activity of vancomycin, amoxicillin, clarithromycin, and azithromycin by human polyclonal IVIG against MDR Gram-positive bacteria, including MRSA, E. faecium, and E. faecalis.

\section{Materials and methods Chemicals}

Unless otherwise indicated, all chemicals were of analytical grade and were purchased from Sigma-Aldrich Co. (St Louis, MO, USA). Prior to the start of the animal experiments, ethical and legal approval was obtained from the Faculty of Pharmacy and Biotechnology, German University in Cairo Committee for Care and Use of Animals for this study. The use of the combined therapy to treat infected mice was approved by the Faculty of Pharmacy and Biotechnology, German University in Cairo Ethics Committee, decision no A-14-2013 for this study.

\section{Antibiotics}

Amoxicillin, oxacillin, and vancomycin were obtained from Sigma-Aldrich Co. Azithromycin was provided by Pfizer, Inc. (New York, NY, USA). Clarithromycin was provided by Abbott Laboratories (Abbott Park, IL, USA.)

\section{Immunoglobulin}

Commercially available human polyclonal IVIG (Gammagard S/D Immune Globulin Intravenous; Baxter International Inc., Deerfield, IL, USA) was supplied as a freeze-dried preparation and was reconstituted in the supplied diluent (Sterile Water for Injection, USP) to $5 \%$ protein $/ \mathrm{mL}$ at an approximate $\mathrm{pH}$ of 6.8 , as described in the manufacturer's manual.

\section{Microorganisms}

Clinical isolates of MRSA $(n=7)$ were provided by the Central Laboratories of the Ministry of Health, Egypt. A reference Staphylococcus aureus strain (ATCC 29213) was obtained from the American Type Culture Collection (ATCC; Manassas, VA, USA). Clinical isolates of E. faecium $(\mathrm{n}=8)$ and E. faecalis $(\mathrm{n}=19)$ were provided by Kasr El Ainy Hospital, Cairo, Egypt. The isolates were identified by using conventional microbiological techniques.

For MRSA, oxacillin susceptibility was tested by inoculation onto a Müeller-Hinton agar plate supplemented with $4 \% \mathrm{NaCl}$ and $6 \mu \mathrm{g} / \mathrm{mL}$ oxacillin, followed by incubation at $37^{\circ} \mathrm{C}$ for 24 hours. The isolates that showed more than one colony were confirmed as MRSA. ${ }^{17}$

The genotypes of the MRSA isolates were examined by pulsed-field gel electrophoresis and analyzed by multilocus sequence typing. These isolates were assigned to clonal complex 8 (CC8), which was found to be the most prevalent MRSA genotype among Egyptian hospitals in epidemiological study conducted in our laboratory (unpublished data). 


\section{Susceptibility of the isolates to the antibiotics}

The minimum inhibitory concentration (MIC) of the antibiotics and the polyclonal IVIG alone was determined by the broth microdilution method using cation-adjusted MüellerHinton broth (MHB) based on the guidelines of the Clinical and Laboratory Standards Institute (CLSI).${ }^{18}$ The minimum bactericidal concentration (MBC) was determined by taking $10 \mu \mathrm{L}$ samples from MIC wells and from wells with higher concentrations and streaking onto the surface of MüllerHinton agar plates. After 24-hour incubation, the number of colony forming units per milliliter $(\mathrm{CFU} / \mathrm{mL})$ was counted and the $\mathrm{MBC}$, defined as the concentration that kills $99.9 \%$ of bacteria, was determined.

\section{Assessment of double combination of the antibiotics with polyclonal IVIG against the isolates using checkerboard assay}

The effectiveness of double combinations of amoxicillin, vancomycin, azithromycin, or clarithromycin with the polyclonal IVIG against isolates of MRSA, E. faecium, and E. faecalis was assessed by checkerboard assay. Because IVIG was found to have no direct antimicrobial activity, the interaction of the combined therapy was assessed with respect to the MICs of the antibiotics. Based on the twofold increase or decrease in the MICs of the antibiotics, the combinatorial response is defined as synergistic, antagonistic, or indifferent. ${ }^{19}$ The interaction type is defined as synergistic (S) if the MIC of the antibiotic decreased by twofold or more compared to its MIC alone. The interaction is indifferent (I) if the MIC of the antibiotic did not change or increased or decreased by onefold concentration in combination. The interaction is antagonistic (A) if the MIC of the antibiotic increased by twofold or more in combination with the polyclonal IVIG.

\section{Evaluation of the double combination of the antibiotics with polyclonal IVIG using time-kill assay}

To verify the results obtained by the checkerboard technique, the bactericidal activity of the antibiotics alone and in combination with the IVIG was determined using the time-kill assay. Ten clinical isolates from the three groups of bacteria were used to assess the antimicrobial activity of the combined therapy. The selected bacteria included seven isolates from combination therapy that showed synergy when the polyclonal IVIG was added to amoxicillin (three isolates, one from each bacterial group), vancomycin (three isolates, one from each bacterial group), or clarithromycin (one isolate of MRSA). The study also included two isolates from combination therapy that showed antagonistic interaction between the antibodies and vancomycin (one MRSA isolate) or clarithromycin (one isolate of E. faecalis). Finally, one isolate of MRSA, which represented indifferent interaction in combination of azithromycin and the IVIG, was also included.

The bacteria were grown in $\mathrm{MHB}$ at $37^{\circ} \mathrm{C}$ until they reached the logarithmic phase. The bacterial suspensions were then centrifuged at $2800 \mathrm{~g}$ for 10 minutes. The cell pellets were washed twice in $10 \mathrm{~mL}$ of normal saline solution. The bacterial suspensions were then used to inoculate $50 \mathrm{~mL}$ MHB containing 10 or $100 \mu \mathrm{g} / \mathrm{mL}$ of IVIG and supplemented with half or one-fourth of the MIC of amoxicillin, azithromycin, clarithromycin, or vancomycin in $250 \mathrm{~mL}$ Erlenmeyer flasks to bring the initial inoculum size to $1 \times 10^{5} \mathrm{CFU} / \mathrm{mL}$. The flasks were incubated in shaking incubator at $37^{\circ} \mathrm{C}$ and $200 \mathrm{rpm}$ for 8 hours. At 2-hour intervals, samples were taken and viable bacterial counts were determined. The experiment was performed in triplicate, and the result was compared to the antibiotics alone and antibiotics-free samples.

\section{Evaluation of the antimicrobial activity of amoxicillin and vancomycin in combination with polyclonal IVIG against invasive MRSA infection in a murine model}

All procedures and guidelines of the German University in Cairo Institutional Animal Care and Use Committee were strictly followed. Male Swiss mice (22-24 g) were obtained and housed five per cage in a biosafety level-2 facility. Standard mouse chow and water were provided. Eight groups each of five mice were used as described in Table 1. MRSA isolate C19 was grown in Tryptic Soy Broth (TSB) medium for 18 hours at $37^{\circ} \mathrm{C}$. Bacterial cell suspensions were centrifuged at $7000 \mathrm{~g}$ for 15 minutes, washed twice in sterile phosphate buffer saline, and finally standardized to $1 \times 10^{8} \mathrm{CFU} / \mathrm{mL}$. The groups of the animal assigned for infection were injected intraperitoneally (IP) with $1 \mathrm{~mL}$ of the bacterial suspension, which led to systemic illness with no death in the animal groups within 5 days after infection. The animals were treated with the antibiotics or the IVIG alone in combination. Two doses of IVIG, $1 \mathrm{~g} / \mathrm{kg}$ and $0.5 \mathrm{~g} /$ $\mathrm{kg}$ body weight, were injected via tail vein at zero time and after 24 hours of infection. Amoxicillin, $7 \mathrm{mg} / \mathrm{kg}$, and vancomycin, $110 \mathrm{mg} / \mathrm{kg}$, were injected IP every 8 hours for 4 days following infection of the animal as previously 
Table I The groups of mice and the corresponding treatment they received

\begin{tabular}{|c|c|c|c|c|c|}
\hline Group & MRSA $\left(\mathrm{I} \times 10^{8} \mathrm{CFU} / \mathrm{mL}\right)$ & Vancomycin & Amoxicillin & IVIG & $5 \%$ albumin \\
\hline I & - & - & - & - & - \\
\hline II & $\mathrm{I} \mathrm{mL}, \mathrm{IP}$ & - & - & - & - \\
\hline III & $\mathrm{I} \mathrm{mL}, \mathrm{IP}$ & 110 mg/kg/8 h & - & - & - \\
\hline IV & $\mathrm{I} \mathrm{mL}, \mathrm{IP}$ & - & $7 \mathrm{mg} / \mathrm{kg} / 8 \mathrm{~h}$ & - & - \\
\hline V & $\mathrm{I} \mathrm{mL}, \mathrm{IP}$ & - & - & $\mathrm{I} \mathrm{g} / \mathrm{kg}, 0.5 \mathrm{~g} / \mathrm{kg}$ & - \\
\hline VI & $\mathrm{I} \mathrm{mL}, \mathrm{IP}$ & - & - & - & $\mathrm{l} g / \mathrm{kg}, 0.5 \mathrm{~g} / \mathrm{kg}$ \\
\hline VII & $\mathrm{I} \mathrm{mL}, \mathrm{IP}$ & $110 \mathrm{mg} / \mathrm{kg} / 8 \mathrm{~h}$ & - & $\mathrm{l} \mathrm{g} / \mathrm{kg}, 0.5 \mathrm{~g} / \mathrm{kg}$ & - \\
\hline VIII & $\mathrm{I} \mathrm{mL}, \mathrm{IP}$ & - & $7 \mathrm{mg} / \mathrm{kg} / 8 \mathrm{~h}$ & $\mathrm{l} \mathrm{g} / \mathrm{kg}, 0.5 \mathrm{~g} / \mathrm{kg}$ & - \\
\hline
\end{tabular}

Note: - stands for no treatment received. Group I, uninfected and received no treatment; group II, infected and received no treatment; group III, infected and treated with vancomycin; group IV, infected and treated with amoxicillin; group V, infected and treated with IVIG; group VI, infected and treated with albumin; group VII, infected and treated with vancomycin and IVIG; group VIII, infected and treated with amoxicillin and IVIG.

Abbreviations: CFU, colony forming units; MRSA, methicillin-resistant Staphylococcus aureus; IVIG, intravenous immunoglobulin G; IP, intraperitoneally; h, hours.

described. ${ }^{20,21}$ All infected animals received $5 \mathrm{mg} / \mathrm{kg}$ of ketoprofen, subcutaneously every 12 hours to manage pain. The control group received two doses of $5 \%$ albumin similar to the IVIG via tail vein. On the fifth day, the mice were euthanized and the kidney, lung, heart, and liver were aseptically collected and homogenized. Briefly, specimens were suspended and homogenized in $5 \mathrm{~mL}$ of pre-cooled normal saline by sonication using Bandelin SONOPULS HD 2070 Ultrasonic Homogenizer, Berlin, Germany, at a continuous sonication cycle set at the maximum output for 30 seconds. One-milliliter samples were then serially diluted in MHB, spread onto nutrient agar plates, and incubated at $37^{\circ} \mathrm{C}$ for 24 hours, and viable colonies were counted.

\section{Statistical analysis}

Each experiment was performed in triplicate, and the mean and standard deviation (SD) were calculated using GraphPad InStat software (GraphPad Software, Inc., La Jolla, CA, USA). One-way analysis of variance (ANOVA) was used to determine the differences between various treatments. Tukey's pairwise comparison test was used with the significance level set at $p<0.05$ to determine significant differences between means.

\section{Results}

\section{Susceptibility of the isolates to the antibiotics}

The antimicrobial efficacy of the antibiotics and the IVIG against the tested isolates was assessed by determination of the MICs and MBCs (Table 2). The IVIG did not show antimicrobial activity when tested alone against the isolates. Vancomycin was effective against the MRSA isolates with an MIC range of $0.25-2.0 \mu \mathrm{g} / \mathrm{mL}$. Resistance to vancomycin was demonstrated in 16 and $25 \%$ of E. faecalis and E. faecium isolates, respectively. Azithromycin and clarithromycin were ineffective against all isolates of MRSA and E. faecalis, while
$74 \%$ of the $E$. faecium isolates were resistant to the antibiotic. All isolates of MRSA and E. faecalis and $87 \%$ of E. faecium isolates were resistant to amoxicillin.

\section{Double combination of the antibiotics with the polyclonal IVIG against the isolates}

The IVIG augmented the antimicrobial activity of the antibiotics against some of the isolates (Figure 1A-C). The outcome of the combination was varied based on the microorganism and the antibiotic tested. Addition of IVIG to amoxicillin showed synergism in $14,12.5$, and $16 \%$ of MRSA, E. faecium, and E. faecalis, respectively, with no antagonism observed. Combination of IVIG and vancomycin, on the other hand, enhanced the antimicrobial activity of the antibiotic with synergism demonstrated in $29,12.5$, and $10.5 \%$ of MRSA, E. faecium, and E. faecalis, respectively. Antagonism was observed in one isolate of MRSA when IVIG was combined with vancomycin.

Combination of IVIG with azithromycin had no effect on the antimicrobial activity of the antibiotic against the isolates (data not shown). Synergism was observed in $29 \%$ of the MRSA when clarithromycin was combined with the antibodies, while the combined therapy showed antagonism in $47 \%$ of $E$. faecalis isolates.

\section{Evaluation of the double combination of the antibiotics with IVIG using time-kill assay}

The time-kill assay was used to verify the results obtained by the broth checkerboard method using ten isolates in which synergistic, antagonistic, or indifferent response was demonstrated. The antibiotics were tested at one-fourth or half of their MICs in combination with the IVIG at 10 or $100 \mu \mathrm{g} / \mathrm{mL}$. MRSA C19, E. faecalis $\mathrm{EF} 4$, and E. faecium AF55 were selected to test the combination of amoxicillin 
Table 2 Susceptibility of the bacterial isolates to the antibiotics

\begin{tabular}{|c|c|c|c|c|}
\hline Microorganism (number) & Amoxicillin, $\mu g / m L$ & Vancomycin, $\mu g / m L$ & Azithromycin, $\mu \mathrm{g} / \mathrm{mL}$ & Clarithromycin, $\mu \mathrm{g} / \mathrm{mL}$ \\
\hline \multicolumn{5}{|l|}{ MRSA (7) } \\
\hline MIC range & $8->32$ & $0.25-2$ & $16 \rightarrow 32$ & $4->32$ \\
\hline $\mathrm{MIC}_{50}$ & 32 & I & $>32$ & $>32$ \\
\hline $\mathrm{MIC}_{90}$ & $>32$ & 2 & $>32$ & $>32$ \\
\hline $\mathrm{MBC}$ range & $8->32$ & $2-32$ & $>32$ & $4->32$ \\
\hline $\mathrm{MBC}_{50}$ & 32 & 16 & $>32$ & $>32$ \\
\hline $\mathrm{MBC}_{90}$ & $>32$ & 32 & $>32$ & $>32$ \\
\hline$\%$ resistance & 100 & 0 & 100 & 100 \\
\hline MIC BP & $\mathrm{iR}$ & $>2$ & $>2$ & $>2$ \\
\hline \multicolumn{5}{|l|}{ E. faecium (8) } \\
\hline MIC range & $8->32$ & $\mathrm{I}->32$ & $>32$ & $>32$ \\
\hline $\mathrm{MIC}_{50}$ & 32 & 2 & $>32$ & $>32$ \\
\hline $\mathrm{MIC}_{90}$ & $>32$ & 4 & $>32$ & $>32$ \\
\hline $\mathrm{MBC}$ range & $32->32$ & $2->32$ & $>32$ & $>32$ \\
\hline $\mathrm{MBC}_{50}$ & $>32$ & 16 & $>32$ & $>32$ \\
\hline $\mathrm{MBC}_{90}$ & $>32$ & $>32$ & $>32$ & $>32$ \\
\hline$\%$ resistance & 87.5 & 25 & 100 & 100 \\
\hline MIC BP & $>8$ & $>4$ & $\mathrm{iR}$ & $\mathrm{iR}$ \\
\hline \multicolumn{5}{|l|}{ E. faecalis (19) } \\
\hline MIC range & $8-32$ & $\mathrm{I} \rightarrow>32$ & $>32$ & $I \rightarrow 32$ \\
\hline $\mathrm{MIC}_{50}$ & 16 & 2 & $>32$ & 16 \\
\hline $\mathrm{MIC}_{90}$ & 32 & 4 & $>32$ & $>32$ \\
\hline $\mathrm{MBC}$ range & $32->32$ & $2->32$ & $>32$ & $16->32$ \\
\hline $\mathrm{MBC}_{50}$ & 32 & 16 & $>32$ & $>32$ \\
\hline $\mathrm{MBC}_{90}$ & 32 & $>32$ & $>32$ & $>32$ \\
\hline$\%$ resistance & 100 & 16 & 100 & 74 \\
\hline MIC BP & $>8$ & $>4$ & $\mathrm{iR}$ & $\mathrm{iR}$ \\
\hline
\end{tabular}

Notes: The $\mathrm{MIC}_{50}$ and $\mathrm{MIC}_{90}$ are the concentration of the antibiotic that inhibits 50 and $90 \%$ of the isolates, respectively. The $M B C_{50}$ and $M B C_{90}$ are the concentration of the antibiotic that kills 50 and $90 \%$ of the isolates, respectively. The microbial resistance was determined based on the MIC BP in $\mu \mathrm{g} / \mathrm{mL}$ according to the European Committee on Antimicrobial Susceptibility Testing. ${ }^{39}$

Abbreviations: MRSA, methicillin-resistant Staphylococcus aureus; MIC, minimum inhibitory concentration; MBC, minimum bactericidal concentration; BP, breakpoint; E. faecium, Enterococcus faecium; iR, intrinsically resistant; $E$. faecalis, Enterococcus faecalis.

with the antibodies because they showed the best synergistic outcomes in the checkerboard assay. The bactericidal activity of amoxicillin at the tested concentrations against the MRSA isolate was significantly enhanced $(p<0.05)$ when the antibiotic at one-fourth or half of its MIC was combined with $100 \mu \mathrm{g} / \mathrm{mL}$ of IVIG (Figure 2). The enhancement was not demonstrated when IVIG was added at $10 \mu \mathrm{g} / \mathrm{mL}$. The synergistic response was also observed against the $E$. faecalis isolate when amoxicillin was combined with $100 \mu \mathrm{g} / \mathrm{mL}$ of IVIG (Figure 3). The enhanced response was demonstrated with the three tested isolates only after 6 or 8 hours of treatment with $100 \mathrm{mg} / \mathrm{mL}$ of IVIG and amoxicillin (Figures 2 and 3).

The interaction was indifferent when the combined therapy, at all tested concentrations, was used against the $E$. faecium isolate.

Indifferent drug interaction was the prevalent outcome when vancomycin, azithromycin, or clarithromycin was combined with the antibodies against MRSA, E. faecalis, and $E$. faecium (data not shown). In all experiments of the time-kill assay, antagonism was not observed even with the combinations that showed antagonism in the checkerboard assay (data not shown).

\section{Effects of polycolonal IVIG on the antimicrobial activity of amoxicillin and vancomycin against invasive MRSA infection in a murine model}

The antimicrobial efficacy of vancomycin and amoxicillin in combination with polyclonal IVIG to prevent MRSA invasion in a murine model was assessed. The IVIG was delivered via tail vein infusion, and MRSA suspension at $1 \times 10^{8} \mathrm{CFU} / \mathrm{mL}$ was injected IP. After various treatments with each antibiotic, the IVIG alone or in combination, the internal organs were isolated and assessed for the bacterial load. The organs showed various bacterial loads, where treatment with amoxicillin was significantly $(p<0.05)$ effective in reducing the number of bacteria compared to the treatments with other antibiotics. The bacterial load in the lung 

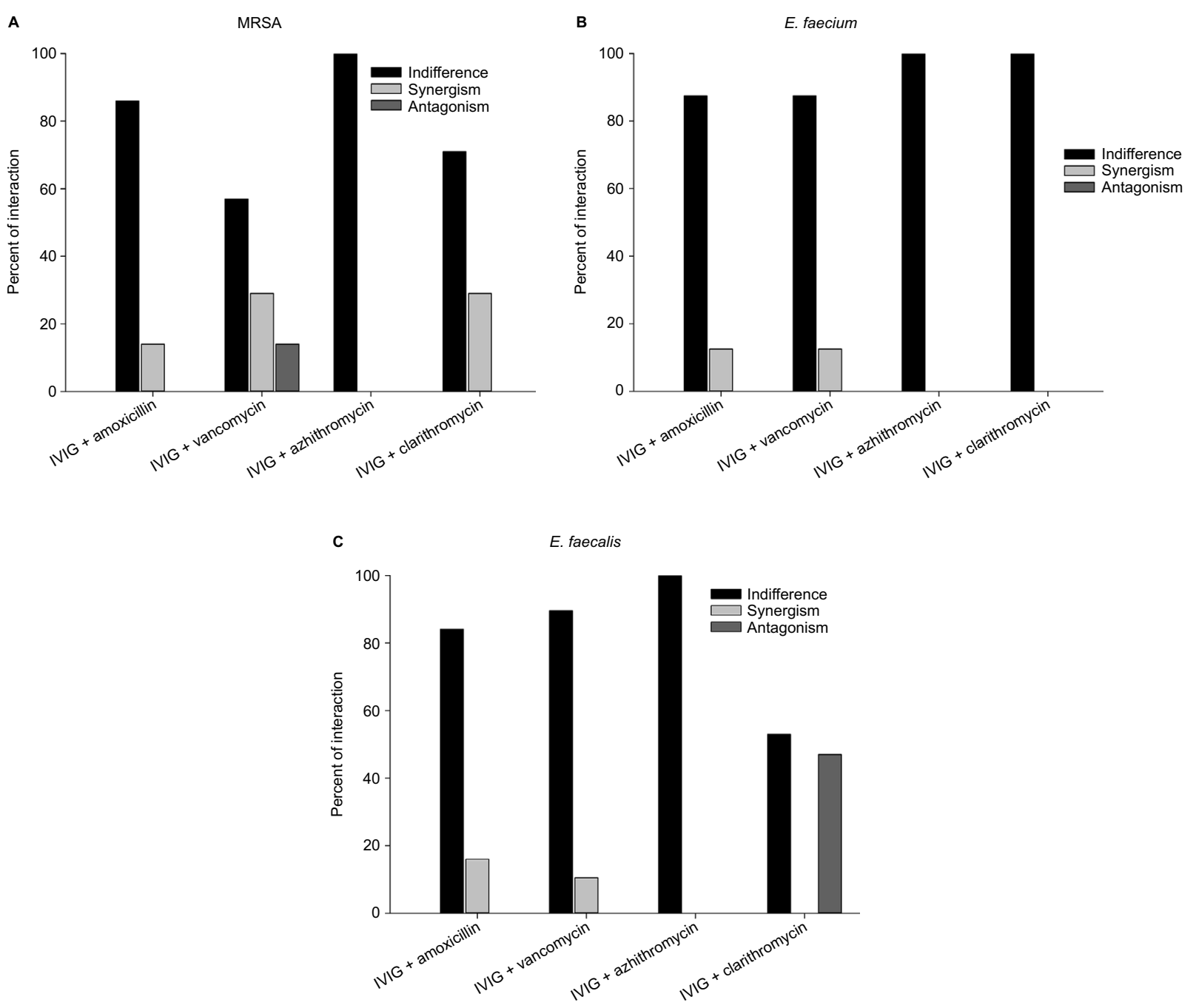

Figure I Double combination of the antibiotics with human polyclonal IVIG against the clinical isolates.

Notes: Checkerboard assay was used to assess the combination of amoxicillin, vancomycin, azithromycin, or clarithromycin with polyclonal IVIG against 34 clinical isolates of MRSA (A), E. faecium (B), and E. faecalis (C). The interaction of the combined therapy was assessed with respect to the MICs of the antibiotics. The interaction is defined as synergistic if the MIC of the antibiotic decreased by twofold or more compared to its MIC alone. The interaction is indifferent if the MIC of the antibiotic remained unchanged, increased, or decreased by onefold concentration in combination. The interaction is antagonistic if the MIC of the antibiotic increased by twofold or more in combination with the polyclonal IVIG.

Abbreviations: IVIG, intravenous immunoglobulin G; MRSA, methicillin-resistant Staphylococcus aureus; E. faecium, Enterococcus faecium; E. faecalis, Enterococcus faecalis; MIC, minimum inhibitory concentration.

was reduced by the IVIG alone, while the antibiotic-IVIG combination therapy failed to prevent dissemination of the bacteria to internal organs (Figure 4).

\section{Discussion}

Owing to the multidrug resistant nature of many pathogens, treatment with conventional antibiotics does not assure desired clinical outcomes. Therefore, new compounds and alternative methods have been investigated to find a new treatment approach. It was proven that combination therapies increase the activity of antibiotics against resistant pathogens. ${ }^{22,23}$ Commercially pooled polyclonal human IVIG is a potential drug that can be used as an antimicrobial agent against infections caused by MDR pathogens. The IVIG has been approved by FDA in primary humoral immunodeficiency and has demonstrated benefits against numerous infections caused by Gram-positive and Gram-negative bacteria. ${ }^{6,11,24}$

IVIG has been shown to possess a complex, dosedependent mechanism of action that varies according to the pathogenesis of the disease. ${ }^{25}$ The drug activity in IgG replacement therapy is explained by the presence of antibodies that have intrinsic capacity to recognize foreign antigens or by the presence of pathogen-specific IgG antibodies resulting from previously immunized or vaccinated serum donors. Its antimicrobial activity is explained by neutralization of the pathogen toxins and superantigens and by preventing them from reaching the target site. Because the drug functions 

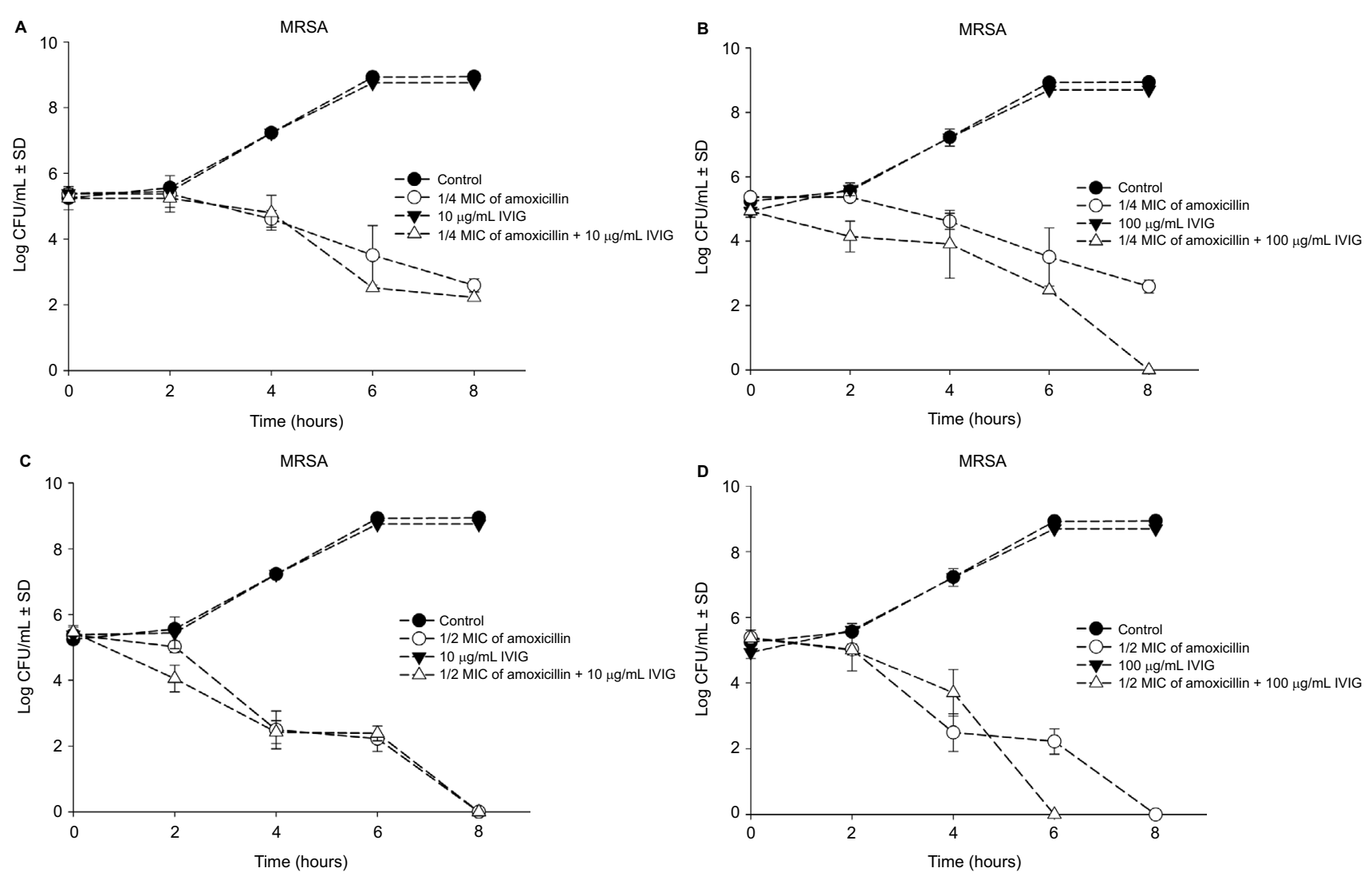

Figure 2 Evaluation of double combination of amoxicillin with polyclonal IVIG against MRSA isolate CI 9 using time-kill assay.

Notes: Isolate $\mathrm{CI} 9$ was selected based on the synergistic response in checkerboard assay when IVIG was combined with amoxicillin $(\mathrm{FIC}=0.125)$. The bacterial suspension at a density of $\mathrm{I} \times 10^{5} \mathrm{CFU} / \mathrm{mL}$ was used to inoculate $50 \mathrm{~mL}$ MHB in $250 \mathrm{~mL}$ Erlenmeyer flasks, before being incubated at $37^{\circ} \mathrm{C}$ under shaking at $200 \mathrm{rpm}$ for 8 hours. The samples were collected at 2-hour intervals, and the viable bacterial counts were determined. A given antibiotic and the polyclonal IVIG were tested individually and in combination using the following four sets of experiments: (A) $10 \mu \mathrm{g} / \mathrm{mL}$ of IVIG and amoxicillin at one-fourth of MIC, (B) $100 \mu g / \mathrm{mL}$ of IVIG and amoxicillin at one-fourth of MIC, (C) $10 \mu \mathrm{g} / \mathrm{mL}$ of IVIG and amoxicillin at half of MIC, and (D) $100 \mu \mathrm{g} / \mathrm{mL}$ of IVIG and amoxicillin at half of MIC.

Abbreviations: IVIG, intravenous immunoglobulin G; MRSA, methicillin-resistant Staphylococcus aureus; FIC, fraction inhibitory concentration; CFU, colony forming units; MHB, Müeller-Hinton broth; MIC, minimum inhibitory concentration; SD, standard deviation.

in an antimicrobial manner distinct from antibiotics, it is improbable that pathogens would develop resistance to it. This suggests that a combination of IVIG and a given antimicrobial agent could possibly help in the treatment of infections caused by MDR pathogens.

In order to assess any potential augmentation of the antimicrobial activity of antibiotics by IVIG, a total of 34 isolates (seven MRSA, eight E. faecium, and 19 E. faecalis) were used in the study. MRSA is one of the major nosocomial pathogens; however, in the past few years, there has been an outbreak of MRSA in populations lacking risk factors for exposure to the health care system. ${ }^{26}$ MRSA is resistant to almost all $\beta$-lactam antibiotics because of a $m e c A$ gene encoding the penicillin-binding protein (PBP) 2 a that has low affinity for $\beta$-lactam antibiotics. ${ }^{27}$ We previously found that members of CC 8 are the prevalent MRSA clones in Egypt (unpublished data).

Enterococci are causative agents for endocarditis and urinary tract, wound, intra-abdominal, and pelvic infections.
Enterococci are among the most common nosocomial organisms. Infection caused by enterococci is endogenous, difficult to treat, and usually requires long courses of antibiotics because of the broad intrinsic and acquired resistance to antimicrobial agents, including glycopeptides (vancomycin and teicoplanin). ${ }^{28}$ Acquired resistance is a result of either mutation or acquisition of new genetic determinants found in plasmid and transposons. $^{28}$

Four antibiotics were chosen for the study: $\beta$-lactam antibiotic (amoxicillin), glycopeptides (vancomycin), and two macrolides (azithromycin and clarithromycin). Clarithromycin and azithromycin have the same mode of action; however, they differ in their spectrum of activity, post-antibiotic effect, dosing, their interaction with other drugs, and their in vitro antimicrobial activity. ${ }^{29-32}$

Our results show the susceptibility of MRSA isolates to vancomycin and their resistance to amoxicillin, azithromycin, and clarithromycin (Table 2). MRSA isolates are, with few exceptions, resistant to all beta-lactam antibiotics and 

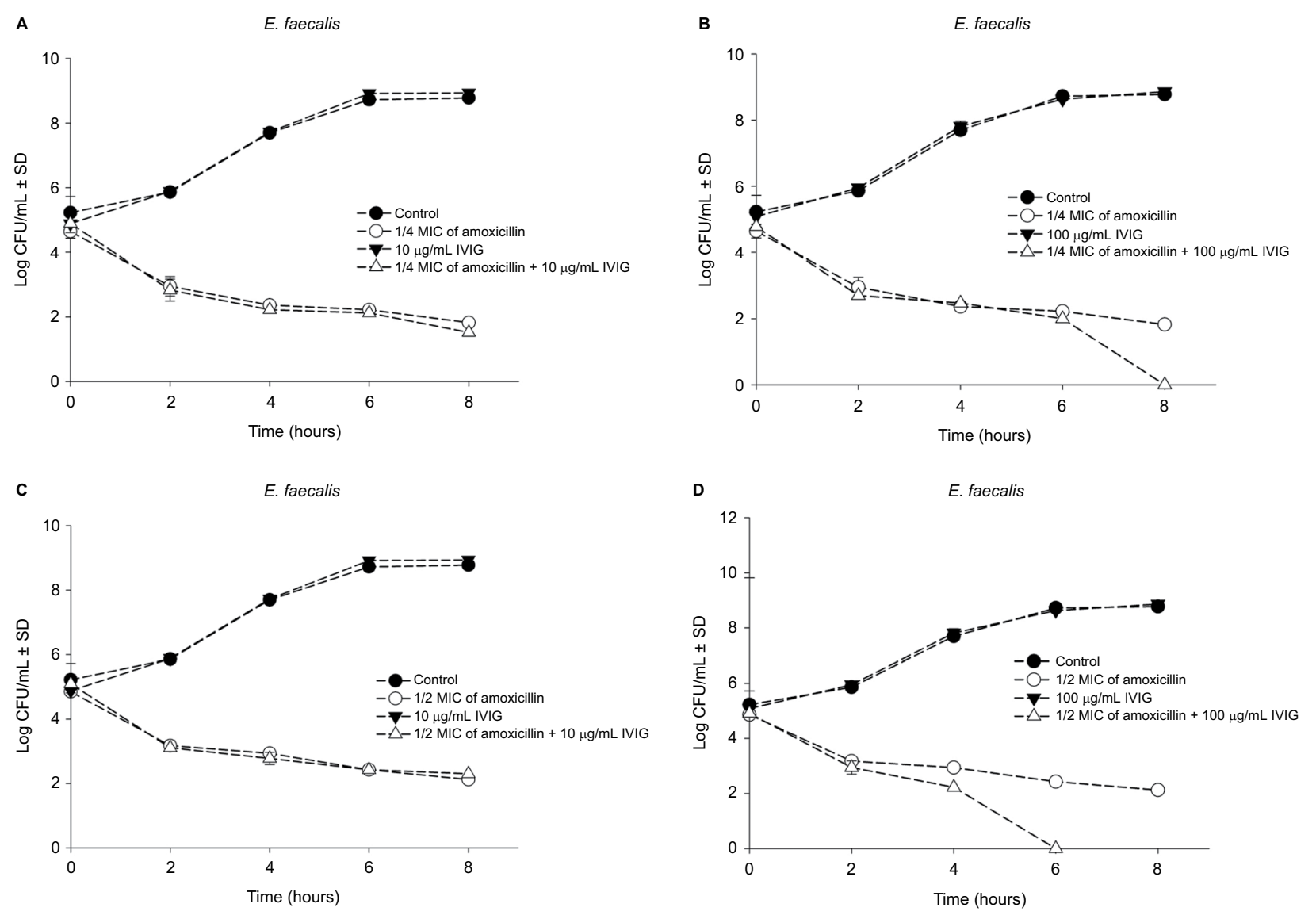

Figure 3 Evaluation of double combination of amoxicillin with polyclonal IVIG against E. faecalis isolate EF4 using time-kill assay.

Notes: Isolate EF4 was selected based on the synergistic response in checkerboard assay when IVIG was combined with amoxicillin (FIC $=0.25)$. A given antibiotic and the polyclonal IVIG were tested individually and in combination using the following four sets of experiments: (A) $10 \mu \mathrm{g} / \mathrm{mL}$ of IVIG and amoxicillin at one-fourth of MIC, (B) $100 \mu \mathrm{g} / \mathrm{mL}$ of IVIG and amoxicillin at one-fourth of MIC, (C) $10 \mu \mathrm{g} / \mathrm{mL}$ of IVIG and amoxicillin at half of MIC, and (D) I00 $\mu \mathrm{g} / \mathrm{mL}$ of IVIG and amoxicillin at half of MIC. Abbreviations: IVIG, intravenous immunoglobulin G; E. faecalis, Enterococcus faecalis; FIC, fraction inhibitory concentration; MIC, minimum inhibitory concentration; SD, standard deviation.

commonly resistant to macrolides, with very rare resistance to glycopeptides antibiotics. ${ }^{22,33}$ The E. faecium and E. faecalis isolates were resistant to amoxicillin and the two macrolides, while 25 and $16 \%$ of the isolates were resistant to vancomycin, respectively. We used antibiotics that are proven to be ineffective against the tested isolates to explore the possibility of enhancement of their antimicrobial activity by IVIG and consequently their reuse against infections caused by MDR bacteria. This approach is very important to face the fast-growing rate of drug resistance with the slow development of new antimicrobial agents.

When checkerboard assay was used to assess the combined therapy, we found that addition of IVIG to amoxicillin, vancomycin, or clarithromycin enhanced the antimicrobial activity of the antibiotics against resistant isolates of MRSA, E. faecium, and E. faecalis, which are intrinsically resistant to the antibiotics (Figure 1A-C). The interaction was indifferent against all isolates when azithromycin was used. Antagonism was demonstrated in one isolate of MRSA in combination of vancomycin with the polyclonal IVIG and in nine isolates of E. faecalis when clarithromycin was tested (Figure 1). The different responses (synergistic, antagonistic, or indifferent) of clinical isolates of the same bacteria to a specific combination would be attributed to phenotypic or genotypic strain variability. The difference could be possibly explained by binding of the IVIG to surface components of the bacteria that would consequently result in increase in the uptake of antibiotics or block of the target sites.

Time-kill assay was used to verify the results obtained by the checkerboard method. Ten isolates, which represent the different types of interaction, were selected. The interaction was considered as synergistic or antagonistic based on the increase or decrease in the $\log _{10}$ value of the viable cell counts by a factor of two or more, respectively. As synergistic drug combinations generate increased efficacy at lower doses, it is more realistic to test the antibiotics at low concentrations to evaluate their effectiveness and efficacy in combination; hence, the antibiotics were tested at subinhibitory concentrations (one-fourth and half of MIC). Synergism was demonstrated when the IVIG was combined with amoxicillin against 


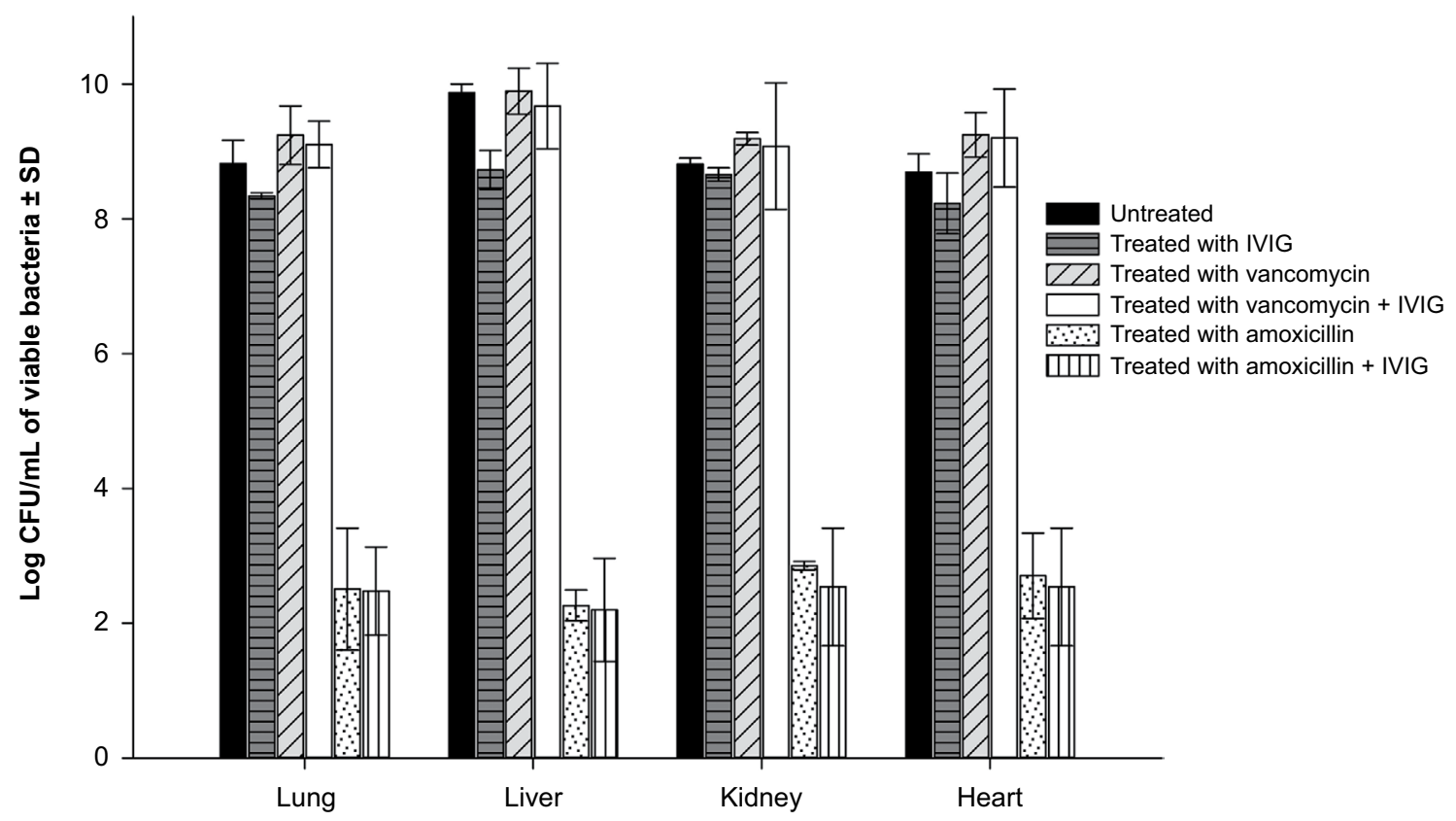

Figure 4 Evaluation of the antimicrobial activity of amoxicillin and vancomycin in combination with polyclonal IVIG against invasive MRSA infection in a murine model. Notes: Eight groups each of five mice were used to assess the combination therapy against invasive sepsis by MRSA isolate CI9. The groups of the animal assigned for infection were injected IP with I mL of the bacterial suspension $\left(I \times 10^{8} \mathrm{CFU} / \mathrm{mL}\right)$. Animals were treated with the antibiotics or the IVIG individually or in combination. On the fifth day post injection, mice were euthanized, the kidney, lung, heart, and liver were aseptically collected and homogenized, and viable bacterial counts were determined. Abbreviations: IVIG, intravenous immunoglobulin G; MRSA, methicillin-resistant Staphylococcus aureus; IP, intraperitoneally; CFU, colony forming units; SD, standard deviation.

MRSA isolate C19 (Figure 2) and E. faecalis isolate EF4 (Figure 3). The killing activity of amoxicillin, at one-fourth or half of MIC, was significantly $(p<0.05)$ enhanced when the polyclonal IVIG was used at $100 \mu \mathrm{g} / \mathrm{mL}$. The interaction between IVIG and the other antibiotics against the tested isolates was indifferent even in the combinations that showed antagonism in the checkerboard assay. The difference in the results obtained by the checkerboard and the time-kill assay could be attributed to the poor correlation of the inhibitory indicator, MIC, with the bactericidal result of the two assays. ${ }^{34}$

The clinical effectiveness of IVIG against bacterial infections has been demonstrated based on data from randomized controlled trials. ${ }^{24}$ Carapetis et $\mathrm{al}^{35}$ conducted a prospective surveillance study in one state of Australia over 2.5 years to determine if there was evidence for IVIG in the treatment of invasive group A streptococcal (iGAS) infection. Their data suggest that the concurrent treatment with IVIG and clindamycin enhanced the treatment of severe iGAS patients.

The efficacy of the combined therapy to prevent invasive MRSA infection in a murine model was investigated. The degree of bacterial dissemination was measured by taking samples from the heart, liver, lungs, and kidneys following infection with MRSA and treatment with amoxicillin or vancomycin alone or in combination with IVIG. The antiinfectivity of polyclonal IVIG alone against MRSA in vivo was previously investigated, where the antibody showed an improvement in the survival of mice infected with MRSA. ${ }^{6}$
In our study, mice were infected by injection of the MRSA isolate C19 IP to cause peritonitis and dissemination to the systemic circulation causing sepsis in the animals. No mortality was observed in any animal groups after 5 days of infection. Following their sacrifice, viable bacterial counts were determined for all treated groups and compared to the bacterial load in the control group. There was no significant effect on the prevention of the bacterial dissemination when IVIG was administered alone or in combination with vancomycin or amoxicillin compared to the effect of each antibiotic. Using a rabbit model of inhalational anthrax, Kammanadiminti et $\mathrm{al}^{36}$ reported that the combination of anthrax-specific immunoglobulin with levofloxacin significantly improved the survival rate of animals.

The insignificant response in our mouse model can be explained by the lower activity of human IVIG in murine blood. ${ }^{6}$ Murine blood has lower neutrophil counts and different - fragment crystallizable receptor (FcR) arsenal on their surfaces. ${ }^{37}$ However, interspecies differences could still affect the activity of the antibodies since human $\mathrm{IgG}$ is known to bind to rodent FcR. ${ }^{38}$ The intravenous injection of the IVIG could have triggered the animal's immune system, leading to its elimination from circulation and consequently preventing it from reaching the peritoneal site where bacteria were introduced. In a previous study, ${ }^{6}$ we used a mouse model to show that IVIG successfully eliminates MRSA when the antibodies were injected IP at the same site of infection. 


\section{Conclusion}

The present study aimed to assess the possible augmentation of the antimicrobial activity of vancomycin, amoxicillin, clarithromycin, and azithromycin by human polyclonal IVIG against MDR Gram-positive bacteria, including MRSA, E. faecium, and E. faecalis.

A checkerboard assay showed that addition of polyclonal IVIG to amoxicillin or clarithromycin enhanced the antimicrobial activity of the antibiotics against isolates of MRSA, E. faecium, and E. faecalis, which are resistant to these antibiotics when used individually. Combination with vancomycin enhanced the efficacy of the antibiotics against $15 \%$ of the tested isolates, while antagonism was observed in one MRSA isolate. Antagonism was also demonstrated in 47\% of the E. faecalis isolates when clarithromycin was tested with polyclonal IVIG. Synergy was proved in time-kill assay when amoxicillin was combined with IVIG against MRSA and E. faecalis, while antagonism was not demonstrated even in all drug combinations that showed this reaction in the checkerboard assay.

The current study is the first to examine a combination approach consisting of human polyclonal IVIG and antibiotics against MDR Gram-positive bacteria in vitro. The combination could be helpful to enhance the antimicrobial activity of not only potent antimicrobial agents but also ineffective antibiotics and would consequently enable their reuse to face the fast-growing drug-resistance with the slow development of new antimicrobial agents.

Further investigation is needed to assess the efficacy of the combination approach on larger numbers of bacteria with wider selection of antibiotics to verify the results obtained by our study.

\section{Disclosure}

The authors declare that none of the submitted materials has any commercial relation and that they do not have conflicts of interest or any source of funding.

\section{References}

1. Carmeli Y, Eliopoulos G, Mozaffari E, Samore M. Health and economic outcomes of vancomycin-resistant enterococci. Arch Intern Med. 2002;162(19):2223-2228.

2. Lodise TP, McKinnon PS. Clinical and economic impact of methicillin resistance in patients with Staphylococcus aureus bacteremia. Diagn Microbiol Infect Dis. 2005;52(2):113-122.

3. Filice GA, Nyman JA, Lexau C, et al. Excess costs and utilization associated with methicillin resistance for patients with Staphylococcus aureus infection. Infect Conrtrol Hosp Epidemiol. 2010;31(4):365-373.

4. Mauldin PD, Salgado CD, Hansen IS, Durup DT, Bosso JA. Attributable hospital cost and length of stay associated with health care-associated infections caused by antibiotic-resistant gram-negative bacteria. Antimicrob Agents Chemother. 2010;54(1):109-115.
5. Giannakaki V, Miyakis S. Novel antimicrobial agents against multidrug-resistant gram-positive bacteria: an overview. Recent Pat Antiinfect Drug Discov. 2012;7(3):182-188.

6. Farag N, Mahran L, Abou-Aisha K, El-Azizi M. Assessment of the efficacy of polyclonal intravenous immunoglobulin G (IVIG) against the infectivity of clinical isolates of methicillin-resistant Staphylococcus aureus (MRSA) in vitro and in vivo. Eur J Clin Microbiol Infect Dis. 2013;32(9):1149-1160.

7. Wisell KT, Kahlmeter G, Giske CG. Trimethoprim and enterococci in urinary tract infections: new perspectives on an old issue. J Antimicrob Chemother. 2008;62(1):35-40.

8. Thouverez M, Talon D, Bertrand X. Control of Enterobacteriaceae producing extended-spectrum beta-lactamase in intensive care units: rectal screening may not be needed in non-epidemic situations. Infect Control Hosp Epidemiol. 2004;25(10):838-841.

9. Hollenbeck BL, Rice LB. Intrinsic and acquired resistance mechanisms in enterococcus. Virulence. 2012;3(5):421-433.

10. Rossi F, Bellon B, Vial MC, Druet P, Kazatchkine MD. Beneficial effect of human therapeutic intravenous immunoglobulins (IVIg) in mercuricchloride-induced autoimmune disease of Brown-Norway rats. Clin Exp Immunol. 1991;84(1):129-133.

11. Barekzi NA, Felts AG, Poelstra KA, Slunt JB, Grainger DW. Locally delivered polyclonal antibodies potentiate intravenous antibiotic efficacy against gram-negative infections. Pharm Res. 2002;19(12): 1801-1807.

12. Darenberg J, Soderquist B, Normark BH, Norrby-Teglund A. Differences in potency of intravenous polyspecific immunoglobulin $\mathrm{G}$ against streptococcal and staphylococcal superantigens: implications for therapy of toxic shock syndrome. Clin Infect Dis. 2004;38(6):836-842.

13. Ishii N, Hashimoto T, Zillikens D, Ludwig RJ. High-dose intravenous immunoglobulin (IVIG) therapy in autoimmune skin blistering diseases. Clin Rev Allerg Immunol. 2009;38(2-3):186-195.

14. Peterlana D, Puccetti A, Simeoni S, Tinazzi E, Corrocher R, Lunardi C. Efficacy of intravenous immunoglobulin in chronic idiopathic pericarditis: report of four cases. Clin Rheumatol. 2005;24(1):18-21.

15. El-Azhary R. Intravenous immunoglobulin in dermatology. $J$ Egypt Womens Dermatol Soc. 2005;2(2):43-46.

16. Norrby-Teglund A, Kaul R, Low DE, et al. Evidence for the presence of streptococcal-superantigen-neutralizing antibodies in normal polyspecific immunoglobulin G. Infect Immun. 1996;46(12):5395-5398.

17. Jonas D, Grundmann H, Hartung D, Daschner FD, Towner KJ. Evaluation of the mecA femB duplex polymerase chain reaction for detection of methicillin resistant Staphylococcus aureus. Eur J Clin Microbiol Infect Dis. 1999;18(9):643-647.

18. Clinical and Laboratory Standards Institute (CLSI). Methods for Dilution Antimicrobial Susceptibility Tests for Bacteria That Grow Aerobically: Approved Standards-Seventh Edition. CLSI Document M7-A7. Vol. 26. Pennsylvania, USA: CLSI; 2006:16-18.

19. El-Azizi M. Novel microdilution method to assess double and triple antibiotic combination therapy in vitro. Intern J Microbiol. 2016;2016:10.

20. Miyazaki S, Fujikawa T, Kobayashi I, Matsumoto T, Tateda K, Yamaguchi $\mathrm{K}$. The in vitro and in vivo antibacterial characterization of vancomycin and linezolid against vancomycin-susceptible and -resistant enterococci. J Antimicrob Chemother. 2002;50(6):971-974.

21. Azoulay-Dupuis E, Moine P, Bedos JP, Rieux V, Vallee E. Amoxicillin dose-effect relationship with Streptococcus pneumoniae in a mouse pneumonia model and roles of in vitro penicillin susceptibilities, autolysis, and tolerance properties of the strains. Antimicrob Agents Chemother. 1996;40(4):941-946.

22. Schwank S, Blaser J. Once- versus thrice-daily netilmicin combined with amoxicillin penicillin, or vancomycin against Enterococcus faecalis in a pharmacodynamic in vitro model. Antimicrob Agents Chemother. 1996;40(10):2258-2261.

23. Aslangul E, Ruimy R, Garry L, Andremont A, Fantin B. Relationship between the level of acquired resistance to gentamicin and synergism with amoxicillin in Enterococcus faecalis. Antimicrob Agents Chemother. 2005;49(10):4144-4148. 
24. Matsuo $\mathrm{H}$, Itoh $\mathrm{H}$, Kitamura $\mathrm{N}$, et al. Intravenous immunoglobulin enhances the killing activity and autophagy of neutrophils isolated from immunocompromised patients against multidrug-resistant bacteria. Biochem Biophys Res Commun. 2015;464(1):94-99.

25. Jolles S, Sewell WA, Misbah SA. Clinical uses of intravenous immunoglobulin. Clin Exp Immunol. 2005;142(1):1-11.

26. David MZ, Daum RS. Community-associated methicillin resistant Staphylococcus aureus: epidemiology and clinical consequences of an emerging epidemic. Clin Microbiol Rev. 2010;23(3):616-687.

27. Neu HC. The crisis in antibiotic resistance. Science. 1992;257(5073): 1064-1073.

28. Ghonaim M, Ghoniem E, Abdulaziz A, Abd Elmotaleb T. Enterococci in hospital associated infection in the national liver institute, Egypt. Egypt J Med Microbiol. 2009;18(3):69-78.

29. Hammerschlag MR, Qumei KK, Roblin PM. In vitro activities of azithromycin, clarithromycin, L-ofloxacin, and other antibiotics against Chlamydia pneumoniae. Antimicrob Agents Chemother. 1992;36(7):1573-1574.

30. Pozzi E, Grossi E, Pecori A. Azithromycin versus clarithromycin in the treatment of acute exacerbations of chronic bronchitis. Curr Ther Res. 1994;55(7):759-764.

31. Arguedas A, Loaiza C, Rodriguez F, Herrera ML, Mohs E. Comparative trial of 3 days of azithromycin versus 10 days of clarithromycin in the treatment of children with acute otitis media with effusion. JChemother. 1997;9(1):44-50.

32. Swanson RN, Lainez-Ventosilla A, De Salvo MC, Dunne MW, Amsden GW. Once-daily azithromycin for 3 days compared with clarithromycin for 10 days for acute exacerbation of chronic bronchitis: a multicenter, double-blind, randomized study. Treat Respir Med. 2005;4(1):31-39.
33. Gorwitz RJ, Jernigan DB, Powers JH, Jernigan JA. Participants in the CDC Convened Experts Meeting on Management of MRSA in the Community. Strategies for Clinical Management of MRSA in the Community: Summary of an Experts' Meeting Convened by the Centers for Disease Control and Prevention; 2006. Available from: http:/www.cdc. gov/ncidod/dhqp/ar_mrsa_ca.html. Accessed July 31, 2016.

34. Cappelletty DM, Michael JR. Comparison of methodologies for synergism testing of drug combinations against resistant strains of Pseudomonas aeruginosa. Antimicrob Agents Chemother. 1996;40(3):677-683.

35. Carapetis JR, Jacoby P, Carville K, Ang SJ, Curtis N, Andrews R. Effectiveness of clindamycin and intravenous immunoglobulin, and risk of disease in contacts, in invasive group a streptococcal infections. Clin Infect Dis. 2014;59(3):358-365.

36. Kammanadiminti S, Patnaikuni RK, Comer J, Meister G, Sinclair $\mathrm{C}$, Kodihalli S. Combination therapy with antibiotics and anthrax immune globulin intravenous (AIGIV) is potentially more effective than antibiotics alone in rabbit model of inhalational anthrax. PLoS One. 2014;9(9):e106393.

37. Sriskandan S, Ferguson M, Elliot V, Faulkner L, Cohen J. Human intravenous immunoglobulin for experimental streptococcal toxic shock: bacterial clearance and modulation of inflammation. J Antimicrob Chemother. 2006;58(1):117-124.

38. Weisman LE, Lorenzetti PM. High intravenous doses of human immune globulin suppress neonatal group B streptococcal immunity in rats. J Pediatr. 1989;115(3):445-450.

39. The European Committee on Antimicrobial Susceptibility Testing (EUCAST) [homepage on the Internet]. Breakpoint Tables for Interpretation of MICs and Zone Diameters. Version 5.0; 2015. Available from: http://www.eucast.org. Accessed November 11, 2016.
Infection and Drug Resistance

\section{Publish your work in this journal}

Infection and Drug Resistance is an international, peer-reviewed openaccess journal that focuses on the optimal treatment of infection (bacterial, fungal and viral) and the development and institution of preventive strategies to minimize the development and spread of resistance. The journal is specifically concerned with the epidemiology of antibiotic

\section{Dovepress}

resistance and the mechanisms of resistance development and diffusion in both hospitals and the community. The manuscript management system is completely online and includes a very quick and fair peerreview system, which is all easy to use. Visit http://www.dovepress.com/ testimonials.php to read real quotes from published authors. 